\title{
Non-transitive Better than Relations and Rational Choice
}

\section{Anders Herlitz ${ }^{1,2}$ (D)}

Received: 16 November 2018 / Revised: 27 February 2019 / Accepted: 13 March 2019 /

Published online: 29 March 2019

(C) The Author(s) 2019

\begin{abstract}
This paper argues that decision problems and money-pump arguments should not be a deciding factor against accepting non-transitive better than relations. If the reasons to accept normative standpoints that entail a non-transitive better than relation are compelling enough, we ought to revise our decision method rather than the normative standpoints. The paper introduces the most common argument in favor of nontransitive better than relations. It then illustrates that there are different ways to reconceptualize rational choice so that rational choice is possible also when the relevant better than relation is non-transitive.
\end{abstract}

Keywords Betterthan relations · Population ethics · Rational choice - Spectrum arguments Transitivity

Perennial problems in population ethics give us some reason to believe that sometimes the most relevant better than relation is not transitive (cf. Parfit 1984; Rachels 1998; Temkin 1987, 2012). This has led some to believe that the all-things-considered better than relation is not transitive (cf. Temkin 1987, 2012). Others reject the relevance of non-transitive better than relations and take it to be a conceptual truth that the all-thingsconsidered better than relation is transitive (cf. Broome 2004). One reason to reject better than relations that are non-transitive is that a transitive better than relation seems required for rational choice to be possible. It has been claimed that views that entail

Anders Herlitz

andersherlitz@gmail.com

1 Institute for Futures Studies, Box 591, S-101 31 Stockholm, Sweden

2 Department of Global Health and Population, Harvard T H Chan School of Public Health, Harvard University, Boston, MA, USA 
non-transitive better than relations are impractical (without transitivity, all alternatives might be worse than some alternative and it is impossible to choose a best alternative) (cf. Handfield 2015). As Jacob N. Nebel puts it:

If transitivity fails, then it may seem impossible for the better than relation to guide our choices or attitudes in many cases. This is because, for any outcome we choose or prefer, there may be some better alternative that we ought to have chosen or preferred instead. The rejection of transitivity might, therefore, lead to skepticism about practical reasoning, or at least about the role of the good in practical reasoning (Nebel 2018: 875).

A different reason to reject better than relations that are non-transitive is that an agent that orders alternatives with such a relation can be money-pumped (explained in further details below) (Huemer 2008; Temkin 2012).

In this paper, I argue that arguments that refer to these problems are not necessarily compelling, and that decision problems and money-pump arguments should not be a deciding factor against accepting non-transitive better than relations (which is not to say that there cannot be other reasons against accepting this). There are ways to modify decision methods and rational choice theory so that also non-transitive better than relations can be action-guiding even when there is no maximal option, and using such modified decision methods also protect against money-pumps.

The argument in the paper makes a new contribution to the debates on Spectrum Arguments in the normative-ethical literature in that it illustrates how non-transitive better than relations can be paired with unconventional conceptions of rational choice in order to form a general view of what it is sensible not to do when some alternatives relate to each other in a cyclical way. I am, thus, not attempting to in this paper provide a general decision method that will enable one to always identify a plausible choice, or even a decision method that helps one identify options that are always all-thingsconsidered reasonable choices. My ambition is much more modest: I will attempt to show that one can accept non-transitive better than relations and adopt a less conventional conception of rational choice in order to determine what are all-things-considered impermissible choices. This means that non-transitive better than relations can be action-guiding even when they entail cyclical evaluations and that the dichotomy between skepticism about action-guidingness and transitivity of the better than relation is false. It is possible - and in my own opinion even probable - that an approach that accepts non-transitive better than relations and unconventional conceptions of rational choice must be amended with some view of how to make all-things-considered reasonable choices after the impermissible alternatives have been discarded, but that is not a topic I will engage with in this paper.

Before presenting the argument, let me point out that there is nothing conceptually mysterious with normative views that generate cyclical orderings of outcomes. It is not difficult to come up with grounds for normative orderings that lead to cycles, and these are perfectly intelligible at a conceptual level (although possibly unacceptable at a substantive level). For example:

Outcome $\mathrm{X}$ is more just than Outcome $\mathrm{Y}={ }_{\operatorname{def}} \mathrm{X}$ is Pareto superior to $\mathrm{Y}$ (i.e. someone is better off, and no one is worse off, in $\mathrm{X}$ compared to $\mathrm{Y}$ ), or if neither 
$\mathrm{X}$ nor $\mathrm{Y}$ is Pareto superior to the other, $\mathrm{X}$ is more equal than $\mathrm{Y}$ as measured with the Gini coefficient (cf. Tungodden and Vallentyne 2005).

Consider how this view would order the following outcomes where each number describes how well off an individual is, the individuals - from left to right - are the same in each outcome, and everything else is equal:

\begin{tabular}{lll}
\hline A & B & C \\
$45 ; 90 ; 100$ & $50 ; 100 ; 200$ & $45 ; 110 ; 90$ \\
\hline
\end{tabular}

B is Pareto superior to A, so B is more just than A. Neither A nor C is Pareto superior to the other, but $\mathrm{A}$ is more equal than $\mathrm{C}$ (Gini coefficient of inequality: $\mathrm{A}$ : 0.156 vs. $\mathrm{B}: 0.177$ ), so $\mathrm{A}$ is more just than $\mathrm{C}$. Neither $\mathrm{B}$ nor $\mathrm{C}$ is Pareto superior to the other, but $\mathrm{C}$ is more equal than $\mathrm{B}$ (Gini coefficient of inequality: C: 0.177 vs. B: 0.286 ), so $\mathrm{C}$ is more just than $\mathrm{B}$. Thus, if we let ' $>$ ' denote more just than:

$$
\mathrm{B}>\mathrm{A} ; \mathrm{A}>\mathrm{C} ; \mathrm{C}>\mathrm{B}
$$

Setting aside disputes concerning the substantive normative ideas that underlie this view, Paretian egalitarianism, some will claim that it is irrational to adopt a normative view that violates transitivity in this way. However, even those who hold that belief will need to accept that these normative views are at least conceptually possible.

The question is thus not whether normative orderings that generate cyclical orderings are possible or not, but whether they are relevant or not, and what reasons we invoke to answer this question. Relevance can mean different things in different contexts. In this paper, I will by relevance mean relevant to determining what one ought to do. I will argue that decision problems and money-pump arguments are not good reasons to dismiss the relevance (in the above sense) of non-transitive better than relations, and suggest that in case the normative standpoints that ground a nontransitive better than relation are compelling enough, it might be better to accept a non-transitive better than relation than to abandon or revise normative beliefs with reference to how they lead to better than relations that are not transitive.

\section{Spectrum Arguments}

The standard argument against transitivity of the better than relation takes the following shape:

Premise 1: Outcomes in a relevant choice set, A-Z, can be ordered on a spectrum where the outcome in one extreme of the spectrum, A, is very good in terms of one of two relevant values dimensions, D1, and relatively bad in terms of the other relevant value dimension, D2, and the outcome in the other extreme of the spectrum, $Z$, is very good in terms of the second relevant value dimension, D2, and very bad in terms of the first relevant value dimension, D1. From A to Y, the outcome that directly succeeds an outcome on the 
spectrum (B for $\mathrm{A}, \mathrm{C}$ for $\mathrm{B} \ldots \mathrm{Z}$ for $\mathrm{Y}$ ) is much better in terms of $\mathrm{D} 2$ and only slightly worse in terms of D1.

Premise 2: An outcome, $x$, is better than another, $y$, in case it is much better in terms of D2, and only slightly worse in terms of D1.

Premise 3: An outcome, $x$, is better than another, $y$, in case it is better in terms of D1, and the difference in D1 is large enough.

Conclusion: The better than relation is not transitive.

The outcomes in the choice set that is described in Premise 1, A-Z, will by the described better than relation be ordered in the following way ('>' denotes 'better than'):

$$
\mathrm{Z}>\mathrm{Y}>\mathrm{X} \ldots \mathrm{B}>\mathrm{A}>\mathrm{Z}
$$

That outcomes next to each other in the spectrum are ordered $\mathrm{Z}>\mathrm{Y}>\mathrm{X} \ldots \mathrm{B}>\mathrm{A}$ follows from Premise 2. That A $>\mathrm{Z}$ follows from Premise 3. Transitivity is violated.

This type of argument, sometimes called 'Spectrum Arguments', has been applied to different areas, and intuitions that support the premises can be found in many fields (cf. Handfield and Rabinowicz 2017; Nebel 2018; Temkin 2012). Population ethicists have applied it to outcomes with populations of different sizes and well-being levels to illustrate how intuitions and widely held normative beliefs in this area seem to support non-transitive better than relations (cf. Parfit 1984; Arrhenius MS). If population B is twice as big as population A, and everyone in population B is only slightly worse off than the people in population A, B is better than A (Premise 2). Yet, a population where everyone has a good life, A, is better than a population where everyone leads lives that are barely worth living, Z (Premise 3). The argument has also been invoked in population-level bioethics to show how intuitions in this area support better than relations that are non-transitive (cf. Herlitz 2018; Norcross 1997; Voorhoeve 2014). If we can choose between treating some group of people with health condition $\mathrm{X}$ or a group of people that is twice as large with health condition $\mathrm{Y}$, and if $\mathrm{X}$ is only a slightly worse health condition, it is better to treat the bigger group from the milder health condition (Premise 2). Yet, it is always better to save a life than to cure headaches, no matter how many headaches we can cure (Premise 3). The argument can be used to study intuitions and normative theories in many other areas as well, for example intuitions and theories about suffering and the complex relations that different kinds of suffering bear to each other (Temkin 2012).

Most writers who engage in these debates believe that the reasons to accept each of the premises are very compelling (cf. Arrhenius et al. 2017; Handfield 2015; Handfield and Rabinowicz 2017; Rachels 1998; Temkin 2012). Yet, people draw very different conclusions from Spectrum Arguments. Whereas some are willing to accept that there is a relevant better than relation that is not transitive, others find the conclusion that the better than relation is non-transitive so implausible so that they conclude that some of the premises of the argument must be false. For example, John Broome has suggested that our intuitions are unreliable when we contemplate large numbers, and that Premise 3 therefore is unsustainable (cf. Broome 2004; Pummer 2013). Others have argued that the argument rather shows that there must be some discontinuity in the spectrum of elements, so that Premise 1 is false and Premise 2 cannot be used to order all outcomes that appear to be next to each other on a spectrum (cf. Dorsey 2009; Parfit 2016). 
Besides strong intuitions, those who reject the notion of non-transitive better than relations can point to how someone who accepts a better than relation that is not transitive can be money-pumped. Consider an illustration. By stipulation, let $x$ be better than $y, y$ better than $z$, and $z$ better than $x$. Money-pump arguments charge that an agent that accepts this will find herself in the following situation: whichever alternative she chooses, she will be willing to pay some money to change her choice to a second alternative, where after she again will be willing to pay some money to go to the third option, and then again pay some money to go back to the first choice. This process can be iterated until the agent has no money left. For instance, if an agent chooses $z$ among the alternatives above, someone might walk up to her and ask her if she would accept changing her mind to $y$ for a small fee. Since $y$ is better than $z$, the agent is supposed to be prepared to pay some fee to change to $y$. Once she has changed her mind, however, someone might walk up to her and ask if she is willing to pay some fee to change her mind from $y$ to $x$. Since $x$ is better than $y$, she is supposed to be willing to make this transaction. And when she has chosen $x$, she will for the same reason be willing to pay some money to go back to $z$. This seems irrational, but it also leads to very undesirable outcomes whenever such a decision method is applied and the circumstances allow for money-pumping.

In the next section, I will suggest that one can accept the general conclusion that there is a relevant better than relation that is not transitive without having to accept that rational choice in light of how options relate to each other is impossible. This means that the cost of accepting this conclusion is smaller than some seem to believe. If the reasons to accept each of the premises are strong enough, the cost of abandoning them is high. If it can be shown that the cost of accepting the relevance of non-transitive better than relations is smaller than previously thought, accepting that some relevant better than relations are not transitive might well be the most reasonable conclusion.

\section{Rational Choice}

Although conventional rational choice theory relies on conventional definitions of the standard positive comparative relations (i.e. better than, worse than and equally as good as) that hold these to be transitive, rational choice is arguably possible also when nontransitive relations are allowed to rank some elements in the choice set. For example, rational choice is arguably possible also when some alternatives are incomparable, which is a non-transitive relation (if $\mathrm{A}$ and $\mathrm{B}$ are incomparable and $\mathrm{A}$ and $\mathrm{C}$ are incomparable, it does not follow that $\mathrm{B}$ and $\mathrm{C}$ are incomparable). Incomparability challenges the possibility of rational choice if one defines a rational choice as a choice that is at least as good as all alternatives (i.e. optimal). However, as for instance Amartya Sen has argued, there is no need to define rational choice in a way that requires optimality (Herlitz 2019; Sen 1997, 2017). One might instead define rational choice so that all alternatives that are not worse than any alternative (i.e. what Sen calls 'maximal') are rational choices (Sen 1997, 2017). With such a definition of rational choice, rational choice is possible also when some alternatives in the choice set are incomparable. Incomparability might entail that no alternative is optimal, but some alternative(s) will still be maximal.

Likewise, there are conceptions of rational choice that can be used when the relevant better than relation is non-transitive. Below, I introduce two such conceptions of 
rational choice. These can both be used to guide decision making when there are cycles in the choice set.

The challenge for rational choice theory when the better than relation is not transitive consists in defining the conditions that separate permissible from impermissible alternatives. In case all elements in the choice set can be ordered with transitive versions of the comparative relations better than, worse than and equally as good as, the conditions of permissibility can be defined in terms of better than or equally as good as all alternatives (optimality). In case all elements in the choice set can be ordered with transitive versions of the comparative relations better than, worse than and equally as good as and incomparability, the conditions of permissibility can be defined in terms of what is not worse than any alternative (maximality). If a non-transitive better than relation orders some elements in the choice set, neither of these conditions of permissibility can be used to guide choice, since it might be the case that no alternative is optimal and no alternative is maximal.

Other views of rational choice are plausible when the better than relation is nontransitive. Consider, first, how rational choice can be conceptualized as Strongly Uncovered choice. Social choice theorists have explored different ways of introducing conditions for considering alternatives impermissible when there are cycles in the choice set with reference to how they are 'covered' (cf. Duggan 2013; McKelvey 1986; Miller 1980). One way of separating permissible from impermissible alternatives when a non-transitive better than relation orders alternatives in the choice set is to refer to how some alternatives might be strongly covered:

Strong Covering: an alternative, $x$, strongly covers another alternative, $y$, in the choice set if and only if it is true that $x>y$ and compared to any other alternative in the choice set, $z$, (a) if $z>x$ then $z>y$, and (b) if $x>z$, then $y>z$.

One might, with reference to this idea of Strong Covering, hold that a rational choice is a choice that is not strongly covered, i.e. a Strongly Uncovered choice. On this view, an alternative, $y$, is irrational in case it is worse than some alternative, $x$, and with respect to every other alternative, $z$, if $z$ is better than $x$ then $z$ is better than $y$, and if $x$ is better than $z$ then $y$ is better than $z$. All alternatives that are not irrational can be held to be rational.

This concept of rational choice can help one make decisions grounded in comparative relations when the relevant better than relation is not transitive. Consider, for example, a choice set that consists of alternatives ordered in the following way:

$$
\mathrm{A}>\mathrm{B} ; \mathrm{B}>\mathrm{C} ; \mathrm{C}>\mathrm{A} ; \mathrm{A}>\mathrm{D} ; \mathrm{D}>\mathrm{B} ; \mathrm{C}>\mathrm{D}
$$

The better than relation that is used to order A-D is clearly not transitive. There is no optimal alternative, and no alternative is maximal. Yet, not all alternatives are strongly uncovered. A strongly covers D. A is better than D, and A and D are related to B and C in the same way. Both A and D are better than B, and both A and D are worse than C. On the view that a rational choice is a strongly uncovered choice, $\mathrm{A}, \mathrm{B}$ and $\mathrm{C}$ are rational choices in the choice set above, whereas $\mathrm{D}$ is not a rational choice.

In population ethics, this idea could be used to explain why an outcome, $x$, that consists of a population that has the same size as an alternative outcome, $y$, and where everyone is worse off than in that alternative outcome with the same size, $y$, is 
impermissible. In population-level bioethics, the idea can be used to explain why an option is impermissible in case there exists an option in the choice set that provides treatment for the same health condition but to a large group of people.

An alternative conceptualization of rational choice that can be used when the relevant better than relation is not transitive takes inspiration from the work of Thomas Schwartz and so-called 'Schwartz sets' (Schwartz 1970, 1972, 1986). Call this a Schwartz choice: an alternative is a Schwartz choice if and only if it is an element in the Schwartz set.

Schwartz set: The Schwartz set is the subset of the set of alternatives in a choice situation that consists of the alternatives made up of the union of all 'Schwartz subsets'. Schwartz subsets are subsets of the set of alternatives in the choice situation such that (1) every element of the subset, $S$, is pairwise unbeaten by every non-element of the subset, and (2) there is no non-empty subset of the subset, $S$, that satisfied the first condition.

This conception of rational choice can also help one make decisions grounded in comparative relations when the relevant better than relation is not transitive. Consider, for example, a choice set that consists of alternatives ordered in the following way:

$$
\mathrm{A}>\mathrm{B} ; \mathrm{B}>\mathrm{C} ; \mathrm{C}>\mathrm{A} ; \mathrm{A}>\mathrm{D} ; \mathrm{B} ; \mathrm{B}>\mathrm{D} ; \mathrm{C}>\mathrm{D}
$$

The better than relation that is used to order A-D is clearly not transitive. There is no optimal alternative, and no alternative is maximal. Yet, not all alternatives are elements in the Schwartz set. Notably, D is in this situation not an element in the Schwartz Set. $\{A, B, C, D\}$ satisfies (1), but so does $\{A, B, C\}$, which means that (2) is not satisfied. $\{A, B, C\}$ is the only subset that satisfies both (1) and (2), and so $\{A, B, C\}$ is the only Schwartz subset and thereby also the Schwartz set. On the view that a rational choice is a Schwartz choice, A, B and C are rational choices in the choice set above, whereas D is not a rational choice.

Both these conceptualizations of rational choice can give sensible advice in some of the cases discussed in the literature, at least relative to some of the competing approaches. First, compare this approach to skepticism, the view that there can be no betterness orderings in these situations. This is by some considered a possible response to problems in population ethics (cf. Arrhenius et al. 2017). The skepticism view gives no advice at all, whereas accepting a non-transitive better than relation and pairing this with a reconceptualization of rational choice can determine that some options are doubtlessly impermissible. Second, compare it to the kind of approaches that present clear answers, for instance total utilitarianism. Views that fully determine an optimal alternative in all situations are overly precise and demand of us to abandon strongly held normative intuitions. Reconceptualizing rational choice so that it permits of conflicting normative intuitions might be more sensible. Those who intuit that Spectrum Arguments are genuinely troublesome ought to accept that a sensible approach is an approach that is not overly precise, but rather allows for a broad range of stances toward these choice situations. Reconceptualizing rational choice in terms of Strongly Uncovered choice or Schwartz choice reflects this.

Conceptualizing rational choice either as a choice of a Strongly Uncovered choice or as a Schwartz choice also means that one cannot be money-pumped in the simplest 
cases at least. The money-pump argument assumes that it is rational to always choose a better alternative over a worse alternative. If one conceptualizes rational choice in terms of being strongly uncovered or a Schwartz choice, it is instead only rational to choose a strongly uncovered option over a covered option and a member of the Schwartz set over an option that is not a member of the Schwartz set. When $x, y$ and $z$ above are all in the choice set, they are all strongly uncovered and they are all members of the Schwartz set. It is on these conceptions of rational choice not rational to change from one strongly uncovered option to another for a small fee, or to change from one member of the Schwartz set to another for a small fee. ${ }^{1}$

The conceptions of rational choice discussed in this section can be both modified and combined in different ways. In this paper, I do not wish to claim that either of them is superior to the other or that they cannot be further developed. The purpose is rather to show that non-transitive better than relations do not necessarily pose a serious challenge to the possibility of rational choice and practical reasoning, that less conventional conceptualizations of rational choice can give some sensible advice in some of the cases discussed in the literature, and that these conceptualizations of rational choice do not expose decision makers to money pumps. Accepting that some relevant better than relations are not transitive is compatible with having an informative conception of rational choice, and this can give sensible advice in choice situation of interest to population ethicists.

It could be objected that although revisions of conceptions of rational choice enable non-transitive better than relations to be somewhat action-guiding, and although this shows how accepting non-transitive better than relations is superior to skepticism in one respect, the position remains unattractive because it does not lead us to the intuitively right judgment in the cases typically discussed by population ethicists and population-level bioethicists. Accepting a cyclical ranking of different alternatives and pairing this with a revision of one's conception of rational choice in the standard Spectrum Arguments will, after all, deem many options permissible (including world $\mathrm{Z}$ in the Repugnant Conclusion and curing headaches in the healthcare example), and this seems wrong. The view will entail that a world with a huge population of people leading lives barely worth living is permissible even if there is an alternative world with a large population of very happy people, and the view will entail that it is permissible to provide minor health benefits to a large group of people even if one could instead save some other person's life. This seems intuitively wrong.

To this objection, one can first point out that there is no view in the literature that renders judgments that are in line with widely held intuitions in all cases. The fact that there are some counterintuitive implications of a view in this field of research is to be expected. Secondly, although the described view has counterintuitive implications

\footnotetext{
${ }^{1}$ Things are different when the choice set changes. If an agent chooses $z$ from the set of alternatives $x, y$ and $z$ in virtue of the fact that it is strongly uncovered/a member of the Schwartz set, and then faces a second choice set that only consists of $y$ and $z$, she will also on these unconventional conceptions of rational choice be willing to pay to change to $y$. This is because $y$ is not strongly uncovered or member of the Schwartz set when $y$ and $z$ are the only options. Thus, although some money-pump arguments can be refuted with these conceptions of rational choice, all versions of money-pump arguments cannot be refuted in this way. However, situations where choice sets need to change in order for money-pumps to work demand more specific circumstances than situations where the choice set does not have to change. These arguments are thus weaker in the sense that they require a more specific kind of circumstances, and they are easier to refute with reference to how the decision maker sees what is in store for them.
} 
these implications must still be considered less counterintuitive than some of the alternative views. Accepting non-transitive better than relations and a revision of the conception of rational choice might imply that both saving a life and providing a vast number of people with very small benefits are permissible. By contrast, total utilitarianism implies that it is only permissible to provide a vast number of people with very small benefits. Those who strongly intuit that it is wrong to choose many small benefits over a very large benefit in cases like these should object more strongly to views that imply that choosing the small benefits is impermissible than to views that imply that both options are permissible. Thirdly, the fact that this view sometimes implies that several alternatives are permissible although none of them is maximal can be seen as a general shortcoming of axiological views. Betterness orderings, one can argue, only brings us so far in this area of ethics. It sometimes leaves us wondering which of many permissible options to choose, and in order to form all-thingsconsidered judgments of what to do we must take other considerations into account; something must complement axiology. It is tenable that this complement can account for why we ought not to actually choose to provide a small number of benefits to a large number of people when we can save a life, even if that option is permissible from an axiological perspective.

Finally, although the view does indeed imply that many alternatives are permissible when the choice set consists of the full spectrum (A-Z), it does significantly better than some alternative views when we do not consider the full spectrums. The view implies that $\mathrm{A}$ is the only permissible choice when only $\mathrm{A}$ and $\mathrm{Z}$ are in the choice set, and $\mathrm{Z}$ is the only permissible choice when only $\mathrm{Y}$ and $\mathrm{Z}$ are in the choice set. This seems sensible. By contrast, total utilitarianism will have to say $\mathrm{Z}$ is better than both $\mathrm{A}$ and $\mathrm{Y}$, while theories that deem A to be better than $\mathrm{Z}$ tend to imply that $\mathrm{Y}$ is better than $\mathrm{Z}$.

\section{Conclusion}

In this paper, I have showed that non-transitive better than relations do not necessarily challenge the possibility of rational choice, and that accepting such relations can help one infer sensible conclusions in certain cases. There are various ways to modify conceptions of rational choice so that one can partition choice sets into permissible and impermissible alternatives also when the elements in the choice set are ordered with a non-transitive better than relation. This has implications for how one ought to respond to Spectrum Arguments and other arguments that indicate that relevant better than relations are not transitive. It gives one less reason to abandon or revise the normative standpoints that entail non-transitive better than relations. If the reasons to accept the normative standpoints are strong enough, it is better to accept the relevance of non-transitive better than relations and focus on developing a conception of rational choice that can be used when the relevant better than relation is not transitive.

Better than relations are based on normative ideas and if arguments that indicate that relevant better than relations are not transitive rely on normative ideas that are considered very plausible, it is problematic to abandon or revise these ideas just because they violate the exigent demands of an idealized view of rationality. If one finds the reasons to accept the normative positions that ground the definition of a nontransitive better than relation compelling, a non-transitive better than relation might be 
the most relevant. If that is the case, the worry that this challenges the possibility of rational choice should not be a reason to revise ones normative standpoints.

This is not to say that the all-things-considered better than relation is non-transitive. It might or it might not be. But the overwhelming uncertainty many experience when it comes to ethical principles might provide reason to accept that the currently most relevant better than relation in certain areas, given our current state of knowledge of ethics, is not transitive.

There might be reasons to reject the relevance of non-transitive better than relations that I have not addressed in this paper. For example, one might have reason to reject the relevance of non-transitive better than relations in case one works in an area in which one needs to use decision methods that require transitive comparative relations. Or there might be reasons to believe that these relations have no place in a certain domain, e.g. when one considers the plausible preferences of a perfectly rational impartial spectator. However, general arguments against the relevance of non-transitive better than relations that refer to the challenge these pose for the possibility of rational choice fail.

Funding This work was supported by The Swedish Research Council, grant number 2017-01382.

\section{Compliance with Ethical Standards}

Conflict of Interest Statement There are no conflicts of interest.

Open Access This article is distributed under the terms of the Creative Commons Attribution 4.0 International License (http://creativecommons.org/licenses/by/4.0/), which permits unrestricted use, distribution, and reproduction in any medium, provided you give appropriate credit to the original author(s) and the source, provide a link to the Creative Commons license, and indicate if changes were made.

\section{References}

Arrhenius, G., Ryberg, J., \& Tännsjö, T. (2017). The repugnant conclusion. In Stanford encyclopedia of philosophy.

Broome, J. (2004). Weighing lives. Oxford: Oxford University Press.

Dorsey, D. (2009). Headaches, lives and value. Utilitas, 21, 36-57.

Duggan, J. (2013). Uncovered sets. Social Choice and Welfare, 41, 489-535.

Handfield, T. (2015). Rational choice and the transitivity of betterness. Philosophy and Phenomenological Research, 89, 584-604.

Herlitz, A. (2018). Committing to priorities: incompleteness in macro-level health care allocation and its implications. The Journal of Medicine and Philosophy: A Forum for Bioethics and Philosophy of Medicine, 43(6), 724-745.

Herlitz, A. (2019). Nondeterminacy, two-step models, and justified choice. Ethics, 129(2), 284-308.

Handfield, T., \& Rabinowicz, W. (2017). Incommensurability and vagueness in spectrum arguments: Options for saving transitivity of betterness. Philosophical Studies, 175, 2373-2387. https://oi. org/10.1007/s11098-017-0963-9.

Huemer, M. (2008). In defence of repugnance. Mind, 117, 899-933.

McKelvey, R. D. (1986). Covering, dominance, and institution-free properties of social choice. American Journal of Political Science, 30, 283-314.

Miller, N. R. (1980). A new solution set for tournaments and majority voting: Further graph-theoretical approaches to the theory of voting. American Journal of Political Science, 24, 68-96. 
Nebel, J. M. (2018). The good, the bad, and the transitivity of Better Than. Nô̂s, 52, 874-899.

Norcross, A. (1997). Comparing harms: Headaches and human lives. Philosophy and Public Affairs, 26, $135-167$.

Parfit, D. (1984). Reasons and person. Oxford: Oxford University Press.

Parfit, D. (2016). Can we avoid the repugnant conclusion? Theoria, 82, 110-127.

Pummer, T. (2013). Intuitions about large number cases. Analysis, 73, 37-46.

Rachels, S. (1998). Counterexamples to the transitivity of better than. Australasian Journal of Philosophy, 76, 71-83.

Schwartz, T. (1970). On the possibility of rational policy evaluation. Theory and Decision, 1, 89-106.

Schwartz, T. (1972). Rationality and the myth of the maximum. Noûs., 6, 97-117.

Schwartz, T. (1986). The logic of collective choice. New York: Columbia University Press.

Sen, A. (1997). Maximization and the act of choice. Econometrica., 65, 745-779.

Sen, A. (2017). Reason and justice: The optimal and the maximal. Philosophy, 92, 5-19.

Temkin, L. (1987). Intransitivity and the mere addition paradox. Philosophy and Public Affairs, 16, $138-187$.

Temkin, L. (1996). A continuum argument for intransitivity. Philosophy and Public Affairs, 25, 175-210.

Temkin, L. (2012). Rethinking the good: Moral ideals and the nature of practical reasoning. Oxford: Oxford University Press.

Tungodden, B., \& Vallentyne, P. (2005). On the possibility of Paretian egalitarianism. The Journal of Philosophy, 102, 126-154.

Voorhoeve, A. (2014). How should we aggregate competing claims? Ethics, 125, 64-87.

Publisher's Note Springer Nature remains neutral with regard to jurisdictional claims in published maps and institutional affiliations. 